\title{
An Enhanced Coding Strategy for FTN-OFDM/OQAM Transceiver Design
}

\author{
Naila Lahbabi*\&, Hao Lin*, Charbel Abdel Nour ${ }^{\&}$, Catherine Douillard ${ }^{\&}$, Pierre Siohan ${ }^{\dagger}$ \\ * Orange Labs, Cesson Sévigné, France. \\ \& Télécom Bretagne, Brest, France. \\ $\dagger$ retired from Orange Labs
}

\begin{abstract}
In this paper, we consider a transmission scheme combining precoded Faster-Than-Nyquist signaling (FTN) and Orthogonal Frequency Division Multiplexing Offset Quadrature Amplitude Modulation (OFDM/OQAM). The precoding method, named Sparse Interference Pre-Cancellation (SIPC), aims at reducing the interference introduced by FTN signaling at the transmitter side. In order to further improve the receiver performance, we propose additional enhancements to the channel encoder, notably, the bit-to-symbol mapping and symbol to timefrequency-positions mapping. Via simulations, we show that the proposed modifications improve the Bit-Error-Rate (BER) performance of the considered transceiver.
\end{abstract}

\section{INTRODUCTION}

Faster Than Nyquist (FTN) signaling is a concept originally introduced by Mazo in 1975 to boost the transmission rate beyond the Nyquist limit. In [1], Mazo stated that it is possible to trade Inter-Symbol Interference (ISI) free transmission for more throughput by overriding the Nyquist criterion [2]. He also showed for Binary Phase Shift Keying (BPSK) that, as long as the boosted transmission rate, i.e., the ratio between Nyquist rate and FTN rate, does not go beyond $1.25 \mathrm{x}$, the resulting minimum sequence distance always keeps constant, provided that the receiver uses Maximum Likelihood Sequence Estimation (MLSE) [3].

Recently, FTN was re-investigated and extended to Orthogonal Frequency Division Multiplex (OFDM) transmissions. In [4], the authors introduced the two dimensional FTN concept which consists in packing signals closer than what is permitted by the Nyquist criterion, in both time and frequency axes. At the receiver side, an iterative receiver based on one dimensional Maximum A posteriori (MAP) decoding and Soft Interference Cancellation (SIC) is proposed.

The previous work on the combination of OFDM/OQAM (or OQAM in short) and FTN, denoted here by FTN-OQAM, was reported in [5], where the classical OQAM was extended to cover FTN transmission mode. To decode FTN signals, an iterative MAP-based receiver, the complexity of which increases with the modulation order, is proposed in [7]. Later, the implementation of FTN-OQAM was revised in [8]. The proposed algorithm is able to approach very closely the promised rate gain of FTN systems without complexity increase compared to a Nyquist system. At the receiver side, a Minimum Mean Square Error Linear Equalization and Interference Cancellation (MMSE LE-IC) was proposed. Unlike the MAP-based receiver, the complexity of the MMSE LE-IC algorithm is independent of the modulation order which makes it a good candidate if high modulation orders are envisaged.

In [9], we have proposed to pre-cancel FTN selfinterference at the transmitter side. To this purpose, we combined a new precoding technique, named Sparse Interference Pre-Cancellation (SIPC), with the transceiver of [8]. The nature of FTN induced interference makes it difficult to jointly precode all the transmitted symbols, even if this interference is known by the transmitter. Therefore, in [9], we proposed to precode only a group of symbols by pre-canceling interference coming from their neighbors. These latter are kept unchanged and contain OFDM/OQAM symbols. At the receiver side, the precoded symbols are first processed and then their soft estimations are used to detect the non-precoded symbols. The proposed precoding makes the iterative-based receiver converge with less iterations.

In this paper, we aim at improving the FTN-OQAM transceiver with SIPC precoding [9] to approach the performance of Nyquist-based systems with better spectral efficiency. First, we propose to modify the channel encoder by using different coding rates for bits composing the precoded and non-precoded symbols, since they are impacted differently by interference. Then, we modify the bit-to-symbol mapping to take into account the different bit-level protection of the Graymapping. Considering the FTN-OQAM time-frequency lattice, FTN self-interference depends on the symbol position. Therefore, we also propose a symbol to time-frequency-positions mapping different from the one used by OQAM. The remainder of the paper is organized as follows. Section II introduces the FTN-OQAM transceiver with SIPC precoding. Section III details the enhanced channel encoder, Bit-to-Symbol mapping and symbol to time-frequency-positions mapping. Simulation results are given in Section IV and Section V concludes the paper.

\section{SIPC FOR FTN-OQAM}

In this section, we briefly present the background of OQAM and the proposed FTN-OQAM transceiver with SIPC. 


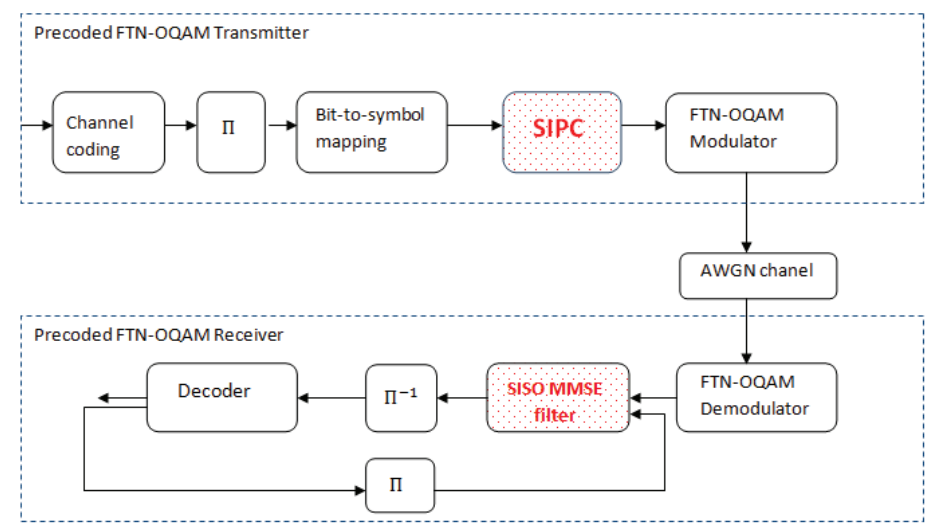

Fig. 1: The proposed precoded FTN-OQAM transceiver in the transmission chain of [8]. Dashed blocks are related to the SIPC method.

\section{A. OQAM}

For $M$ subcarriers, the continuous-time OQAM baseband signal is [6]:

$$
s(t)=\sum_{m=0}^{M-1} \sum_{n=-\infty}^{+\infty} a_{m, n} \underbrace{g\left(t-\frac{n T_{0}}{2}\right) e^{j 2 \pi m F_{0} t} e^{j \Phi_{m, n}}}_{g_{m, n}(t)} .
$$

Pulses $g_{m, n}$ are called basis functions and construct a Hilbert basis, with $T_{0} F_{0}=1$, where $T_{0}$ is the symbol duration and $F_{0}$ the subcarrier spacing. $g(t)$ is a prototype function of length $L$ (without any restriction, we suppose $L=b M, b \in \mathbb{N}$ ) and $\Phi_{m, n}$ is a phase term often equal to $\frac{\pi}{2}(m+n)+\Phi_{0}$, where $\Phi_{0}=0$ or $\pm \pi m n$. The real-valued transmitted symbol $a_{m, n}$ on the $m^{t h}$ subcarrier at time instant $n$ is obtained by taking the real and imaginary parts of the complex-valued $2^{2 K}$ QAM constellation. The orthogonality constraint of OQAM is expressed using the real inner product as:

$$
\Re\left\{\int_{-\infty}^{+\infty} g_{m, n}(t) g_{m^{\prime}, n^{\prime}}^{*}(t) d t\right\}=\delta_{m, m^{\prime}} \delta_{n, n^{\prime}},
$$

where, $\delta_{m, n}$ is the Kronecker symbol. Note that this orthogonality condition can only be satisfied at the Nyquist rate $T_{0} F_{0}=1$ or above.

\section{B. SIPC precoding}

Considering FTN signaling with OQAM, the modulated baseband signal at the FTN-OQAM modulator output is [8]:

$$
s(t)=\sum_{m=0}^{M-1} \sum_{n=-\infty}^{+\infty} a_{m, n} g\left(t-n \tau \frac{T_{0}}{2}\right) e^{j 2 \pi m F_{0} t} e^{j \Phi_{m, n}},
$$

where $0<\tau<1$ is the FTN time packing factor. Its discrete version is:

$$
s[k]=\sum_{m=0}^{M-1} \sum_{n \in \mathbb{Z}} a_{m, n} g\left[k-n N_{f}\right] e^{\frac{j 2 \pi m\left(k-\frac{D}{2}\right)}{M}} e^{j \Phi_{m, n}}
$$

where, $T_{s}=\frac{T_{0}}{M}$ is the sampling rate, $F_{0}=\frac{1}{M T_{s}}, M$ is the size of IFFT/FFT and $D=L-1$.
$N_{f}$ is called the FTN factor and is defined as $N_{f}=\left\lfloor\tau \cdot \frac{M}{2}\right\rfloor$, where $\lfloor$.$\rfloor denotes the floor function.$

Since orthogonality constraint no longer holds, FTN introduces Inter-Symbol Interference (ISI) and Inter-Carrier Interference (ICI). Even if this interference is known by the transmitter, it is difficult to pre-cancel it from all transmitted symbols. In [9], it is proposed to precode only a group of symbols by removing the contribution of their neighbors, while the rest ones are not precoded and remain normal constellations. Precoded symbols are expressed as:

$$
\begin{aligned}
c_{m, n}=a_{m, n}-\alpha \underbrace{\left(\sum_{q=-l, q \neq 0}^{l} h_{q} a_{m, n+q}\right)}_{I S I} & \underbrace{\left.\sum_{p=-l^{\prime}, p \neq 0}^{l^{\prime}} \sum_{q=-l}^{l} g_{p, q, n} a_{m+p, n+q}\right)}_{I C I_{n}},
\end{aligned}
$$

$h_{q}$ and $g_{p, q, n}$ are the coefficients of the equivalent channel of $I S I$ and $I C I_{n}$, respectively. $\left(l, l^{\prime}\right)$ defines the zone of neighboring symbols contributing to ISI and ICI, respectively. The factors $(0 \leq \alpha, \beta \leq 1)$ are introduced to control the amount of pre-canceled interference. Note that FTN self-interference depends on time instance $n$. According to $\alpha$ and $\beta$, three varieties of SIPC precoders are proposed. SIPC at time axis (SIPC-t) consists in pre-canceling ISI $(\beta=0)$. SIPC at frequency axis (SIPC-f), consists in pre-canceling ICI $(\alpha=0)$. Finally, SIPC at both time and frequency axes (SIPC-tf), aims at pre-canceling both ISI and ICI.

The receiver employs a two-stage process based on the Soft-In-Soft-Out (SISO) MMSE LE-IC presented in [8]. First, the precoded symbols are equalized and decoded. Their interference with the non-precoded symbols is then predicted, using the feedback from the decoder. Last, the non-precoded symbols are processed. Thanks to this precoding technique, 
it is shown that the receiver converges with less iterations compared to FTN-OQAM transceiver without precoding [8].

Due to SIPC method, the precoded and non-precoded symbols are impacted differently by the interference. Consequently, we propose to use different coding rates to encode these symbols. We also consider the different bit-level protection of Gray mapping and modify the bit-to-symbol mapping. Finally, since interference level depends on $n$, we introduce a symbol to time-frequency-positions mapping different from what is usually used by OQAM.

\section{OPTIMIZATION OF THE SIPC-T PRECODER}

In this section, we consider the precoded FTN-OQAM transceiver of [9] and propose to modify the channel encoder, the bit-to-symbol mapping and the symbol to time-frequencypositions mapping.

\section{A. Channel coding enhancement}

In Fig. 1, the channel coding block encodes $N$ information bits and delivers $B$ coded bits. We consider that the first $N_{1}$ bits constitute the precoded symbols and the remaining $N_{2}$ bits the non-precoded ones. We propose to use different coding rates $r_{1}$ and $r_{2}$ to encode the $N_{1}$ and $N_{2}$ bits, respectively. Note that $N_{1}$ and $N_{2}$ are chosen as multiples of $r_{1}$ and $r_{2}$, respectively. Without any restriction, we suppose $r_{1} \leq r_{2}$ and replace the channel coding and $\Pi$ blocks by the structure depicted in Fig. 2. The first group of $N_{1}$ bits is encoded and the resulting $B_{1}$ bits are then punctured by block Punct $_{1}$, resulting in a group of $B_{1}^{\prime}=\frac{N_{1}}{r_{1}}$ bits. These bits are then interleaved. Similarly, the second group of $N_{2}$ bits is encoded and punctured, resulting in a group of $B_{2}^{\prime}=\frac{N_{2}}{r_{2}}$ bits, which are then interleaved. Finally, these two group of bits are concatenated to obtain $B=B_{1}^{\prime}+B_{2}^{\prime}$ coded bits. Although several local coding rates are used, the overall initial coding rate remains unchanged.

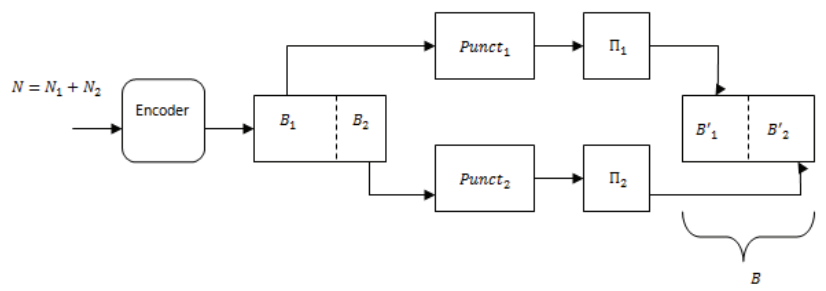

Fig. 2: The proposed channel encoder and interleaver.

\section{B. Bit-to-Symbol mapping enhancement}

Considering a high-order modulation like 64-QAM with Gray-mapping, it is known that the level of protection of each bit of the 6-bit constellation symbol depends on its position. For 64-QAM, three protection levels are available. Thus, we propose to reorder the coded bits at the channel coding output, in Fig.1, in order to map the systematic bits to the positions in the Gray-mapping with the best protection. To this end, we propose to interleave the systematic and parity bits separately. Then, for each 6-bit symbol, the systematic bits are mapped to the three best protected positions, while the remaining positions contain the corresponding parity bits. The same idea applies if 16-QAM modulation is envisaged. Since, 16-QAM offers two different protection levels, the systematic bits are mapped to the two better protected positions while the corresponding parity bits are mapped to the other positions.

\section{Enhancement of the mapping of symbol to time-frequency positions}

In Fig.1, coded bits are interleaved, mapped to complex symbols and then to time-frequency positions by alternating the real and imaginary parts as indicated by OQAM modulation. As mentioned earlier, the transmitted symbols are impacted differently by interference. Therefore, we propose to map symbols made of systematic bits to positions least affected by interference and symbols made of parity bits to the remaining positions.

To this end, we replace blocks $\Pi$ and bit-to-symbol mapping by the scheme illustrated in Fig. 3. The Channel coding block delivers $B=B_{1}^{\prime}+B_{2}^{\prime}$ coded bits, with $B_{1}^{\prime}$ and $B_{2}^{\prime}$ the group of bits composing the precoded and non-precoded symbols, respectively. Sets $S_{1}$ and $S_{2}$ contain the systematic bits of the $B_{1}^{\prime}$ and $B_{2}^{\prime}$ coded bits, respectively. Similarly, sets $P_{1}$ and $P_{2}$ contain the parity bits of the $B_{1}^{\prime}$ and $B_{2}^{\prime}$ coded bits, respectively. First, as interference is known at the transmitter side, we compute its power at the precoded symbols positions. These positions are then sorted in ascending order of their interference power. Set $I_{1}$ contains the first half of these positions, while set $J_{1}$ contains the other half. Finally, precoded symbols obtained from interleaved bits in $S_{1}$ are mapped to positions in $I_{1}$, while precoded symbols obtained from interleaved bits in $P_{1}$ are mapped to positions in $J_{1}$. Similarly, sets $I_{2}$ and $J_{2}$ are constructed according to the interference power at the non-precoded symbols positions. Non-precoded symbols obtained from interleaved systematic bits in $S_{2}$ are mapped to positions in $I_{2}$, while non-precoded symbols obtained from interleaved bits in $P_{2}$ are mapped to positions in $J_{2}$.

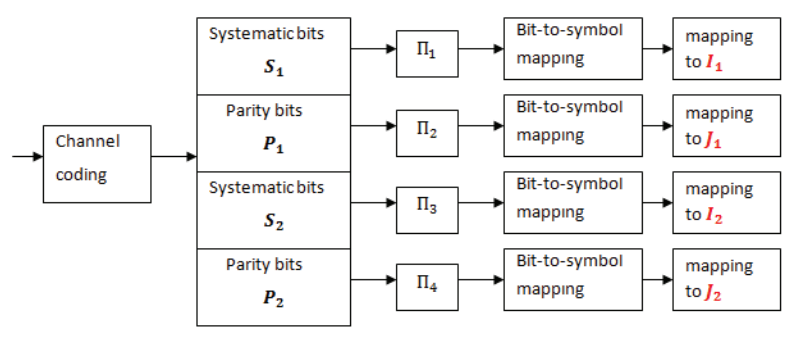

Fig. 3: The proposed symbol to time-frequency-positions mapping. 


\section{Simulation RESUlTs}

In this section, we evaluate the BER performance of our enhanced precoded FTN-OQAM transceiver using SIPC-t precoding as an example.

In our simulations, we used a $(1,5 / 7)$ Recursive Systematic Convolutional (RSC) code of rate $\frac{1}{2}$ for channel coding with random interleaving, 16-QAM and 64-QAM Gray-mapping, a MAP-based outer decoder and an MMSE filter of length 30 and delay 15 . We consider that all the $M=64$ subcarriers are to be modulated. As with SIPC-t we only target ISI cancellation, we have selected two frequency selective prototype filters in order to limit the ICI impact. We used a Square Root Raised Cosine filter with a roll-off factor of 0.5 SRRC (RO=0.5) and PHYDYAS filter [12], both of length $4 M$. In (5) we set $(l=2)$. Note that the number of iterations necessary for the receiver to converge varies according to the modulation order and the packing factor $\tau$. Hence, according to each simulation case, results will be given after having performed the corresponding number of iterations at the receiver side.

Fig. 4 evaluates the BER performance of the FTN-OQAM transceiver with enhanced SIPC-t precoding using PHYDYAS filter, 64-QAM and $\tau=0.8$, after having performed 7 iterations at the receiver side. The red curve with diamonds represents the BER of Nyquist-based OQAM transmission with the modified Gray-mapping. Bit Mapp Op and Symb Mapp Op stand for Bit-to-Symbol mapping and Symbol to time-frequencypositions optimization. In this case, modifying the symbol to time-frequency-positions mapping gives the best results in terms of BER.

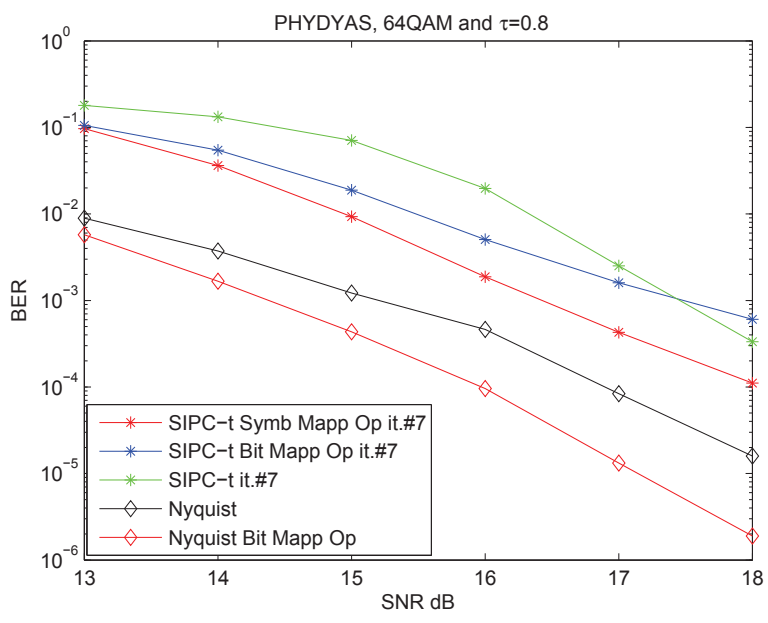

Fig. 4: BER evaluation for SIPC-t with enhanced Graymapping and symbol to time-frequency-positions mapping.

Fig. 5 shows the BER curve, after performing 5 iterations at the receiver side, in case of 64-QAM with SRRC $(\mathrm{RO}=0.5)$ and $\tau=0.9$. For this configuration, SIPC-t with the modified Gray-mapping gives better performance than the modified symbol-mapping. Moreover, the BER curve converges to the Nyquist-based OQAM curve with the modified Gray-mapping. Therefore, the proposed symbol-mapping is advantageous for low packing factors $\tau$ leading to high interference levels, while the proposed bit-mapping is advantageous for high $\tau$.

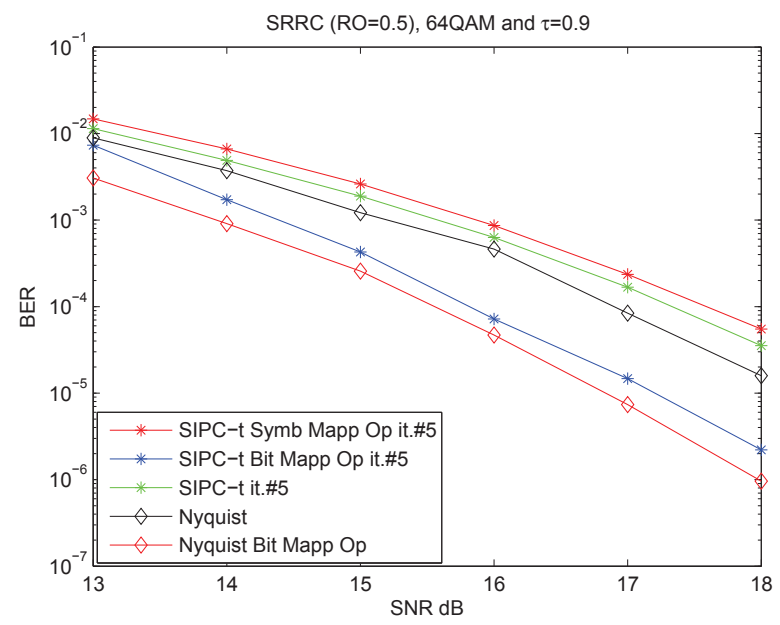

Fig. 5: BER evaluation for SIPC-t with enhanced Graymapping and symbol to time-frequency-positions mapping.

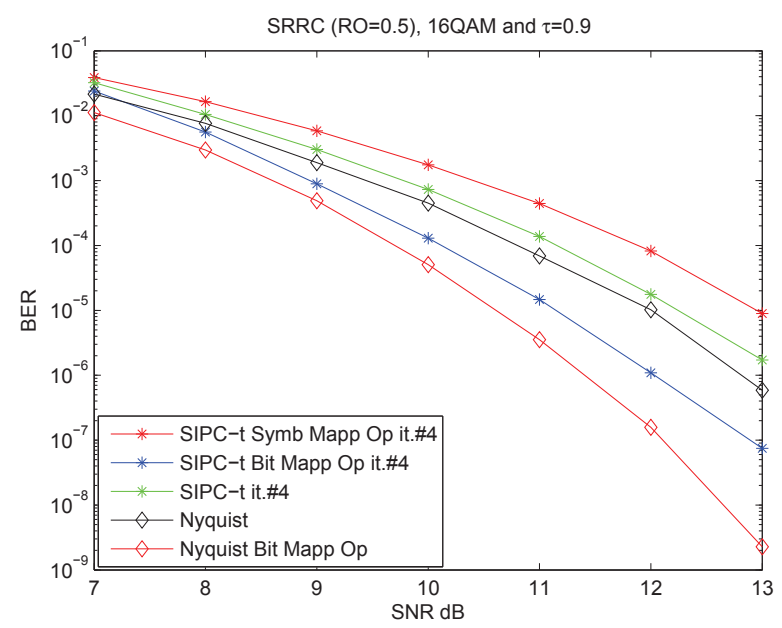

Fig. 6: BER evaluation for SIPC-t with enhanced Graymapping and symbol to time-frequency-positions mapping.

The same tendency applies when 16-QAM modulation is considered. Fig. 6 shows that, for SRRC $(\mathrm{RO}=0.5)$ filter and $\tau=0.9$, the proposed bit-to-symbol mapping improves the SIPC-t performance. As for 64-QAM case, for low packing factors $(\tau=0.7)$, results not reported here, the proposed Symbol-mapping gives better results while the proposed Bitmapping is advantageous for high packing factors $(\tau=$ $0.8,0.9)$.

In what follows, we evaluate the BER performance of SIPC$t$ precoding with the enhanced channel encoder. The mother 
coding rate of the convolutional code is equal to $r=\frac{1}{4}$. The desired overall rate is $\frac{1}{2}$. We consider $\left(r_{1}, r_{2}\right)=\left(\frac{8}{17}, \frac{9}{17}\right)$ to encode the bits composing the precoded and non-precoded symbols, as described in Fig. 2, respectively. In Fig. 7, we consider a 64-QAM modulation with PHYDYAS filter and $\tau=0.8$. The black curves with asterisks and diamonds correspond to SIPC-t at iteration 9 and Nyquist-based OQAM, respectively. These curves are obtained using the same convolutional code and puncturing to ensure a rate of $\frac{1}{2}$. The red curve represents the BER of SIPC-t with the proposed channel encoding. The green and blue curves represent the BER of SIPC-t with the proposed channel encoder combined with the proposed Bit-mapping and Symbol-mapping, respectively. We can observe that SIPC-t with the modified channel encoder has better performance than SIPC-t precoding. As in the previous paragraph, the proposed channel encoder combined with the modified symbol-mapping performs better for $\tau=0.8$ and at high SNR (where the interference becomes the most critical) than when combined with the proposed Bit-mapping.

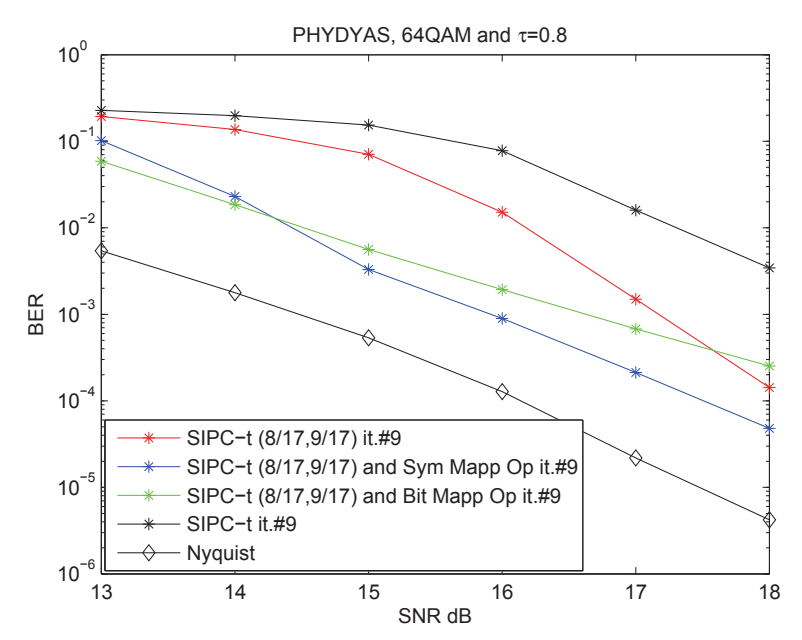

Fig. 7: BER evaluation for SIPC-t, $\tau=0.8$, with enhanced channel coding, Gray-mapping and symbol to time-frequencypositions mapping.

Fig. 8 keeps the same configurations and compares the BER performance of the same schemes when 64-QAM, SRRC ( $\mathrm{RO}=0.5$ ) and $\tau=0.9$ are considered. In this configuration, SIPC-t with the enhanced channel coding converges to the same BER performance as SICP-t after performing 4 iterations at the receiver side. However, considering the BER performance after 3 iterations, the proposed channel coding enables more than $1 \mathrm{~dB}$ gain for an error rate around $10^{-3}$. Moreover, further improvements are obtained when combining the enhanced channel coding with the proposed bit-mapping: after performing 4 iterations at the receiver side, the BER curve converges to the Nyquist performance.

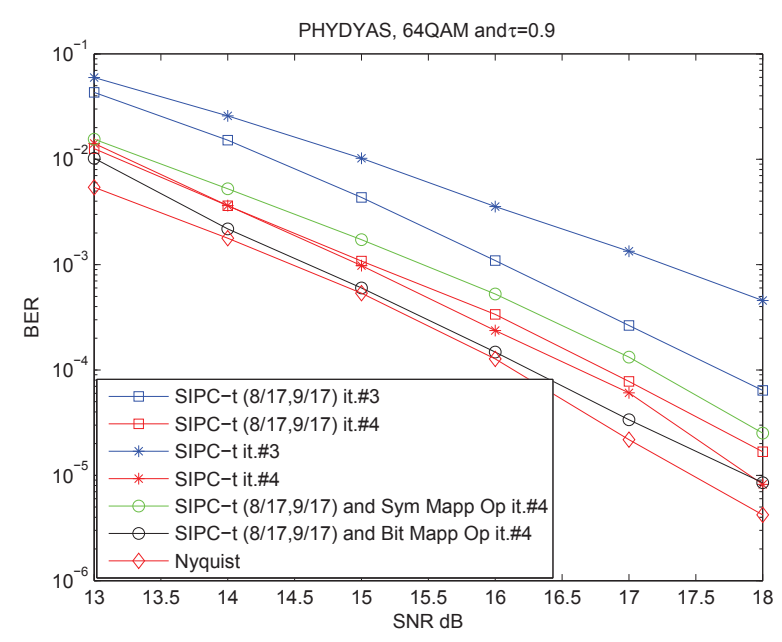

Fig. 8: BER evaluation for SIPC-t, $\tau=0.9$, with enhanced channel coding, Gray-mapping and symbol to time-frequencypositions mapping.

\section{CONCLUSiON}

In this paper, we considered an FTN-OQAM transceiver with a precoding method named Sparse Interference PreCancellation (SIPC). The SIPC method aims at precoding a group of symbols by pre-canceling interference coming from their neighbors while keeping these latter unchanged. This precoding technique allows the iterative-based receiver to converge with less iterations. In order to further improve the receiver performance, we proposed to modify the channel encoder, the Gray-mapping and the symbol to time-frequencypositions mapping. Our objective is to take into account the different bit-level and symbol-level protection against interference. Via simulations, we showed that the proposed bitmapping and symbol-mapping improve the performance of the considered FTN-OQAM transceiver. We also showed that is advantageous to modify the symbol-mapping for low $\tau$ and the bit-mapping for high $\tau$. Moreover, when puncturing is considered to ensure a certain rate, we showed that using different coding rates to encode the precoded and non-precoded symbols can improve the receiver performance.

\section{REFERENCES}

[1] E. Mazo. "Faster-Than-Nyquist signaling”. Bell. Syst. Tech. Journal, 54:1451-1462, 1975.

[2] H. Nyquist. "Certain topics in telegraph transmission theory".Trans. AIEE, vol. 47, pp.617-644, Apr. 1928.

[3] G. Forney. "Maximum-likelihood sequence estimation of digital sequences in the presence of intersymbol interference". Information Theory, IEEE Trans, vol. 18, no. 3, pp. 363-378, 1972.

[4] J.B. Anderson and F. Rusek. "Improving OFDM: Multistream FasterThan-Nyquist Signaling”. In Turbo Codes Related Topics; 6th International ITG-Conference on Source and Channel Coding (TURBOCODING), 2006 4th International Symposium, pp. 1-5, Apr. 2006.

[5] D. Dasalukunte, F. Rusek and J.B. Anderson. "Transmitter architecture for Faster-Than-Nyquist signaling systems". In Circuits and Systems, 2009. ISCAS 2009. IEEE International Symposium, pp. 1028-1031, May. 2009. 
[6] P. Siohan, C. Siclet and N. Lacaille. "Analysis and design of OFDM/OQAM systems based on filterbank theory”. IEEE Trans. Signal Process, vol. 50, no. 5, pp. 1170-1183, 2002.

[7] D. Dasalukunte, F. Rusek, V. Owall. “An Iterative Decoder for Multicarrier Faster-Than-Nyquist Signaling Systems”. In Communication (ICC) 2010. IEEE International Conference, pp. 1-5, May. 2010.

[8] H. Lin, N. Lahbabi, P. Siohan and X. Jiang. "An efficient FTN Imple mentation of the OFDM/OQAM System”. In Communication (ICC), 2015. IEEE International Conference, pp. 4787-4792, June. 2015

[9] N. Lahbabi, H. Lin, C. Abdel Nour, C. Douillard and P. Siohan. “ Sparse
Interference Pre-Cancellation for FTN-OQAM Systems". In European Wireless (EW), 2016. IEEE International Conference, May, 2016.

[10] "Physical Layer for Dynamic Spectrum Access and Cognetive Radio". http://www.ict-phydyas.org/index.php/camax/page/view?id=21.

[11] F. Rusek and J.B. Anderson. "Constrained Capacities for Faster-ThanNyquist Signaling”. Information Theory, IEEE Trans, vol. 55, no. 2, pp. 764-775, Feb. 2009

[12] M. Bellanger. "Specification and design of a prototype filter for filter bank multicarrier transmission". IEEE International Conference on Acoustics, Speech, and Signal Processing (ICASSP), 2001. 